\title{
High NUCB2 expression level is associated with metastasis and may promote tumor progression in colorectal cancer
}

\author{
JUN XIE $^{1,2}$, LINA CHEN ${ }^{3}$ and WENBIN CHEN ${ }^{1}$ \\ ${ }^{1}$ Department of Colorectal Surgery, The First Affiliated Hospital, Zhejiang University School of Medicine, \\ Hangzhou, Zhejiang 310003; Departments of ${ }^{2}$ Colorectal Surgery and ${ }^{3}$ Paediatrics, Affiliated Hospital of \\ Shaoxing University, Shaoxing, Zhejiang 312000, P.R. China
}

Received August 7, 2017; Accepted February 5, 2018

DOI: $10.3892 / \mathrm{ol} .2018 .8523$

\begin{abstract}
Nucleobindin 2 (NUCB2) is mainly expressed in the hypothalamic nuclei and has a proven role in energy homeostasis. It has also been recently reported to have a key role in tumor progression. However, the clinical significance of NUCB2 in colorectal cancer (CRC) remains unknown. In the present study, the level of NUCB2 mRNA was quantified by reverse transcription-quantitative polymerase chain reaction (RT-qPCR) in 34 paired fresh tissues from patients with CRC. RT-qPCR was followed by immunohistochemical (IHC) staining of NUCB2 protein in tissue microarrays of 251 samples to evaluate the clinical significance of NUCB2 in CRC. The RT-qPCR indicated an upregulation of NUCB2 mRNA in CRC tissues compared with normal tissues $(\mathrm{P}=0.027)$. IHC staining indicated a positive association between elevated NUCB2 expression and lymph node metastasis or tumor-node-metastasis (TNM) stage. Patients with CRC and lymph node metastasis demonstrated a higher expression of NUCB2 $(49.5 \%, 50 / 101)$ compared with those without lymph node metastasis $(36.7 \%, 55 / 150 ; \mathrm{P}=0.043)$. Furthermore, NUCB2 expression was also higher in patients with CRC and TNM stage III-IV compared with those with TNM stage I-II (50.9\% vs. $35.0 \%$; $\mathrm{P}=0.011)$. However, Kaplan-Meier analysis indicated no significant association between NUCB2 expression and disease-free survival of patients. Additionally, multivariate analysis did not identify the upregulation of NUCB2 as an independent prognostic predictor in patients with $\mathrm{CRC}(\mathrm{P}=0.755)$. In conclusion, the present study demonstrated that upregulation of NUCB2 is significantly associated with CRC metastasis, indicating that
\end{abstract}

Correspondence to: Dr Wenbin Chen and Dr Jun Xie, Department of Colorectal Surgery, The First Affiliated Hospital, Zhejiang University School of Medicine, 79 Qingchun Road, Hangzhou, Zhejiang 310003, P.R. China

E-mail: cwbin@hotmail.com

E-mail: xjinsx@yahoo.com

Key words: nucleobindin 2, metastasis, cancer progression, colorectal cancer
NUCB2 may be a cancer-associated oncogene associated with the aggressive progression of CRC.

\section{Introduction}

Colorectal cancer (CRC) is the third most commonly diagnosed cancer type, and it is also the third highest cause of cancer-associated mortalities globally (1). Of all cases of CRC, $\sim 20-25 \%$ are metastatic and $50-60 \%$ of the remainder eventually develop metastases as per statistical estimation $(2,3)$. Therefore, it is important to identify novel CRC biomarkers to enhance the efficiency of early diagnosis and improve therapeutic strategies.

Nucleobindin 2 (NUCB2), a precursor of the hypothalamic neuropeptide nesfatin-1, is mainly expressed in the hypothalamic nuclei, and it has specific roles in energy homeostasis (4). It is reportedly distributed in the central nervous system, gastrointestinal system, reproductive organs and adipose tissue (5). Additionally, NUCB2 has been indicated to serve an important role in cancer progression and metastasis (6-10). High levels of NUCB2 mRNA and protein are associated with shorter recurrence-free survival time in prostate cancer $(6,7)$ and increased migration of prostate cancer cells compared with low expression of NUCB2 (8). High expression of NUCB2 is also associated with metastasis and reduced overall survival in clear cell renal cell carcinoma (9). In addition, NUCB2 has been reported to be a potent prognostic factor for primary breast carcinoma as it is associated with its metastasis (10).

These findings strongly indicate that NUCB2 could serve a vital role in inducing metastasis in various types of cancer. However, a number of studies have also demonstrated that NUCB2 inhibited the proliferation of human adrenocortical carcinoma and ovarian epithelial carcinoma cells $(11,12)$. These conflicting results point towards a possible tissue-specific regulatory function of NUCB2. Kan et al (8) indicated that NUCB2 promoted the migration, invasion and epithelial-mesenchymal transition (EMT) CRC cells in vitro and in vivo. Nevertheless, the exact underlying mechanism of NUCB2's action in CRC remains unclear. In the present study, the expression levels of NUCB2 mRNA and protein were analyzed in CRC tissues and adjacent non-cancerous tissues via reverse transcription-quantitation polymerase chain reaction (RT-qPCR) and immunohistochemistry (IHC), 
respectively. The association between NUCB2 expression and the clinicopathological parameters of CRC was also evaluated to determine its clinical significance.

It was predicted that utilizing a larger sample size would generate more consistent data that would help improve the understanding of the role of NUCB2 in the pathological progression of CRC.

\section{Materials and methods}

Patients and tissue samples. The project was approved by the Ethics Committee of the First Affiliated Hospital, Zhejiang University School of Medicine (Hangzhou, China), and each patient was required to provide written informed consent. Samples of cancerous and adjacent non-cancerous colorectal tissue were collected between January 2010 and December 2010 from 34 patients (age range 45-68 years, mean age 55.7 years), comprising of 19 males and 15 females) with CRC admitted to the First Affiliated Hospital, Zhejiang University School of Medicine.

Tissue microarrays (TMA) with 251 CRC paraffinembedded specimens were purchased from Shanghai Biochip Co. Ltd. (Shanghai, China), and were used for detection of NUCB2 expression by IHC staining. The TMAs included samples from 138 males and 112 females, and 1 of unknown sex, with a median age of 66 years (range, 27-91) at the time of operation. Tumor node metastasis (TNM) stage was classified using AJCC cancer staging manual (13). All patients were followed-up for $>5$ years following surgery, and the survival time was calculated from the date of surgery until the deadline for follow-up, or until the date of mortality. An additional 30 samples of normal colorectal tissues were also collected from patients with CRC between January 2014 and December 2014 (16 males and 14 females, age range 38-70 years, mean age 58.6 years) at the First Affiliated Hospital, Zhejiang University School of Medicine (Zhejiang, China).

$R T$ - $q P C R$. Total RNA was extracted from fresh tissues using TRIzol ${ }^{\circledR}$ (Invitrogen; Thermo Fisher Scientific, Inc., Waltham, MA, USA) and the concentration was determined using Nanodrop ${ }^{\mathrm{TM}} 2000$ spectrophotometer (Thermo Fisher Scientific, Inc.). The mRNA samples were stored at $-80^{\circ} \mathrm{C}$. RT-qPCR was performed with iTaq $^{\mathrm{TM}}$ Universal One-Step RT-qPCR kit (Bio-Rad Laboratories Inc., Hercules, CA, USA) according to the manufacturer's protocol. The NUCB2 primers used were: Forward, 5'-TCTTGGAGCCAGATAGCTGG-3' and reverse, 5'-AGCTTCTGAGCCTCCAGTTG-3'. GAPDH was used as an internal control with the following primers: Forward, 5'-TGAAGGTCGGAGTCAACGG-3' and reverse, 5'-CTGGAAGATGGTGATGGGATT-3'. All primers were purchased from Sangon Biotech Co., Ltd. (Shanghai, China). The following cycling conditions were used: $10 \mathrm{~min}$ at $50^{\circ} \mathrm{C}$ for cDNA synthesis plus $1 \mathrm{~min}$ at $95^{\circ} \mathrm{C}$ for reverse transcription inactivation and Taq polymerase activation, followed by 35 cycles of $10 \mathrm{sec}$ at $95^{\circ} \mathrm{C}$ denaturation and $30 \mathrm{sec}$ at $58^{\circ} \mathrm{C}$ for extension and data collection. The NUCB2 mRNA level was calculated relative to the internal GAPDH control with the $\Delta \Delta \mathrm{Cq}$ method (14). For each sample, the PCR was run in duplicates every time and was repeated for a total of three times.
Haematoxylin and eosin staining. Sections were stained with haematoxylin and eosin (H\&E) prior to IHC staining. First, sections were de-paraffinized with xylene, rehydrated in a graded alcohol series (100, 95 and 75\%), and then washed briefly in distilled water and stained with hematoxylin for $8 \mathrm{~min}$ at room temperature. Following this, the sections were washed in running tap water for $5 \mathrm{~min}$ and differentiated in $1 \%$ acid alcohol for $30 \mathrm{sec}$ at room temperature. The sections were washed in running tap water for $5 \mathrm{~min}$ and counterstained in eosin-phloxine solution for $30 \mathrm{sec}$ to $1 \mathrm{~min}$ at room temperature. Then the sections were dehydrated and mounted.

IHC staining. Tissue were fixed by $10 \%$ formaldehyde for $24 \mathrm{~h}$ at room temperature, and TMA sections was cut to 3-4 $\mu \mathrm{m}$ thicknesses and then used for IHC. Briefly, TMA sections were first de-paraffinized with xylene, rehydrated in graded alcohol (100, 95, 85 and $75 \%$ at room temperature) and autoclaved for $3 \mathrm{~min}$ in $0.01 \mathrm{M}$ citrate buffer ( $\mathrm{pH}$ 6.0) for antigen retrieval. The sections were then incubated with $3 \%$ (v/v) $\mathrm{H}_{2} \mathrm{O}_{2}$ for $10 \mathrm{~min}$ at room temperature in order to block endogenous peroxidase. This was followed by incubation with $10 \%$ (v/v) normal goat serum (Thermo Fisher Scientific, Inc.) for $15 \mathrm{~min}$ at room temperature to reduce nonspecific binding. Subsequently, the slides were incubated overnight with rabbit polyclonal antibody against human NUCB2 (dilution, 1:1,000; catalog no. HPA008395; Sigma-Aldrich, Merck $\mathrm{KGaA}$, Darmstadt, Germany) at $4^{\circ} \mathrm{C}$. Following rinsing with phosphate-buffered saline (PBS) three times, the sections were incubated with biotin-labeled secondary antibody contained within the Histostain-Plus kit (HRP, Broad Spectrum) for IHC staining (ready-to-use, 859043; Thermo Fisher Scientific, Inc.) at room temperature for $20 \mathrm{~min}$, followed by horseradish peroxidase-conjugated goat anti-rat antibody (ready-to-use, 859043; Thermo Fisher Scientific, Inc.) for an additional $20 \mathrm{~min}$ at room temperature. Finally, the sections were stained with 3,3-diaminobenzidine for $3 \mathrm{~min}$ at room temperature, counterstained with hematoxylin, dehydrated with graded alcohol $(75,85,95$ and $100 \%)$ and then mounted. For the negative control, PBS was used instead of the primary antibody.

Evaluation of IHC staining. The degree of immunostaining was semi-quantitatively evaluated by two independent expert pathologists who were blinded to the clinical data. The pathologists scored number of positively stained cells per field under a light microscope and viewed five fields under x200 magnification. The level of NUCB2 expression was calculated on the basis of the intensity of staining and the percentage of positively stained cells. The staining intensity was graded according to the following criteria: 0, no staining; 1, weak staining; 2 , moderate staining; and 3, strong staining. To determine the percentage of stained cells, the number of stained and unstained cells was counted per field under x 200 magnification, and the average of five fields was calculated. Based on the percentage of positive cells, the tumor score was graded as follows: $0, \leq 5 \%$ positively stained tumor cells; $1,6-25 \%$ positive tumor cells; $2,26-50 \%$ positive tumor cells; and $3, \geq 51 \%$ positive tumor cells. The staining index was calculated by multiplying the staining intensity score 

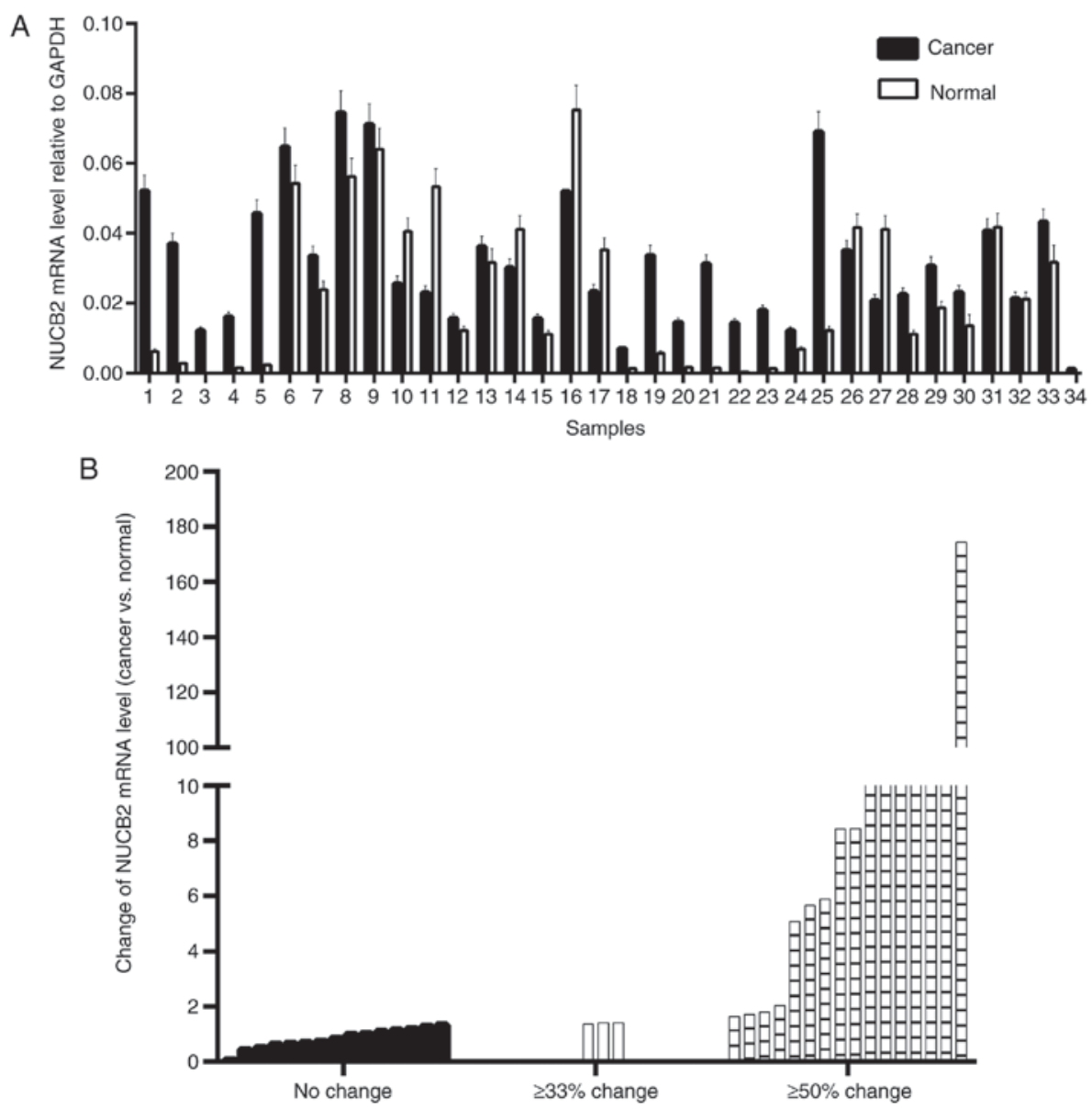

Figure 1. Gene expression of NUCB2 in CRC and adjacent normal tissues. (A) The relative mRNA levels of NUCB2 normalized to GAPDH in 34 paired specimens. (B) NUCB2 mRNA level normalized to GAPDH in CRC compared with normal tissues. CRC, colorectal cancer; NUCB2, nucleobindin 2.

with the percentage of positive cells. For the final evaluation, a staining index score of $\leq 3$ was defined as low NUCB2 expression, and a staining index score of $\geq 4$ was defined as high NUCB2 expression.

NUCB2 staining in the tumor cells was also quantified as integrated optical density (IOD) by estimating the area of the objects and medium pixel intensity per object using the Image-Pro Plus software version 6 (Media Cybernetics, Inc., Rockville, MD, USA).

Statistical analysis. SPSS (version 13.0; SPSS, Inc., Chicago, IL, USA) was used to perform all statistical analyses. For quantitative values with normal distribution, the paired-sample Student's t-test was used for comparison, and if the values were not distributed normally, the Wilcoxon Sign Rank test was used to compare two groups of paired values. $\chi^{2}$ or Fisher's exact test was used to analyze categorical data and evaluate the associations between the expression of NUCB2 and the clinicopathological parameters of CRC. The Kaplan-Meier method was used to perform univariate survival analysis, and the log-rank test was used to calculate differences between the survival curves. Multivariate survival analysis and Cox proportional hazards regression model were used to assess predictors associated with prognosis. $\mathrm{P}<0.05$ was considered to indicate a statistically significant difference.

\section{Results}

Detection of NUCB2 mRNA expression level. To detect NUCB2 mRNA expression level, a total of 34 paired fresh $\mathrm{CRC}$ specimens and their surrounding normal mucosal tissues were analyzed using RT-qPCR. NUCB2 upregulation was defined when the NUCB2 mRNA level was higher in the cancer tissue compared with the non-cancerous tissue from the same patient. Conversely, downregulation of NUCB2 was defined when a lower NUCB2 expression was detected in the cancer tissue compared with the non-cancerous tissue.

NUCB2 mRNA levels were upregulated in $73.5 \%$ of CRC (25/34) samples and downregulated in the remaining $26.5 \%$ of samples (9/34) (Fig. 1A). Paired Student's t-test demonstrated that the mean level of NUCB2 mRNA was upregulated in CRC tissues compared with the normal tissues $(\mathrm{P}=0.018)$. Depending on the extent of the relative change in NUCB2 mRNA level in the cancer tissues, the samples were divided into three groups (no change, $\geq 33 \%$ change and $\geq 50 \%$ change) and nearly half of the samples indicated $\mathrm{a} \geq 50 \%$ change (Fig. 1B).

Association of NUCB2 expression with clinicopathological features of CRC. The presence and distribution of NUCB2 in the tissues was assessed via IHC staining. The NUCB2 protein was primarily localized in the cytoplasm and, to a lesser extent, in the membrane of the cancer cells (Fig. 2). High 
expression of NUCB2 was detected in 5/30 (16.7\%) normal tissue samples, and in 105/251 (41.8\%) CRC tissue samples, indicating a significant difference $\left(\chi^{2}=7.124, \mathrm{P}=0.008\right)$.

In order to assess the association between NUCB2 expression and colon cancer progression, the clinicopathological parameters of colon cancer was analyzed in samples that exhibited high levels of NUCB2. The results indicated a positive association between NUCB2 expression and lymph node metastasis and TNM stage (13) (Table I). Patients with lymph node metastasis had a higher expression of NUCB2 (49.5\%, 50/101) compared with those without lymph node metastasis (36.7\%, 55/150; $\mathrm{P}=0.043)$. In addition, patients with TNM stage III-IV exhibited significantly higher NUCB2 expression compared with those with TNM stage I-II $(50.9 \%$ vs. $35.0 \%$; $\mathrm{P}=0.011$ ). The quantification of NUCB2 staining also indicated that the IOD values in tumors with lymph node metastasis and TNM stage III-IV were higher compared with those in tumors without lymph node metastasis and TNM stage I-II (Fig. 3; $\mathrm{P}<0.05)$.

Clinical significance of NUCB2 expression in prognosis of $C R C$. Univariate survival analysis demonstrated that the 3 - and 5 -year cumulative survival rates of patients with high expression of NUCB2 were 64.3 and $52.0 \%$, and the 3- and 5-year cumulative survival rates were 71.9 and $61.6 \%$ in those with no NUCB2 expression, respectively. The mean survival duration of patients with CRC with a high NUCB2 expression was $56.27 \pm 3.42$ months, and the mean survival duration of patients with low NUCB2 expression was $62.59 \pm 2.79$ months (Fig. 4). In addition, the analysis indicated no significant association of NUCB2 expression with overall survival (Fig. $4 ; \chi^{2}=2.044$, $\mathrm{P}=0.153)$.

\section{Discussion}

CRC is one of the most frequently occurring cancer types globally (15). Metastasis in patients with colon cancer is associated with poor prognosis at early stages of the disease and subsequent mortality (16). Therefore, it is vital to investigate the underlying molecular mechanisms of metastasis and develop therapeutic strategies specifically targeting this process (17). NUCB2 has a widespread expression pattern in the body and mainly participates in physiological processes, including nocturnal feeding and regulation of body weight (18). Recent reports have demonstrated the diverse functions of NUCB2 in various tissues and in different cancer types $(11-13,19)$. Takagi et al (19) reported that NUCB2 expression was positively correlated with Ki67 expression, and the knockdown of NUCB2 significantly inhibited the proliferation and migration of tumor cells in endometrial carcinoma. By contrast, NUCB2 also inhibited cell proliferation in adrenocortical and ovarian epithelial carcinoma $(11,12)$. Notably, Kan et al (8) determined that NUCB2 enhanced migration, invasion and EMT of cancer cells in colon cancer. Nevertheless, the clinical significance of NUCB2 in colon cancer remains unclear.

In the present study, RT-qPCR indicated an upregulation of NUCB2 mRNA levels in CRC tissues compared with the adjacent non-cancerous tissues, which was consistent with previous reports on prostate cancer $(6,20)$. The association of NUCB2 protein expression with CRC progression was further

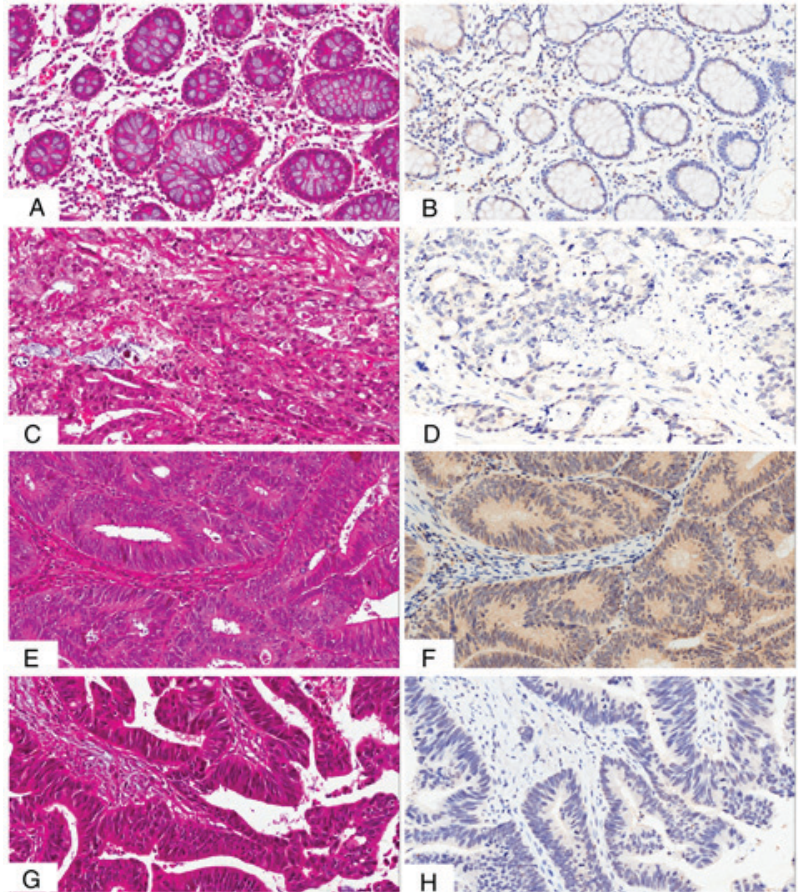

Figure 2. IHC staining of NUCB2 in CRC tissues. (A) H\&E staining of low expression of NUCB2 in normal tissues. (B) Low expression of NUCB2 in normal tissues. (C) H\&E staining of low NUCB2 expression in poorly differentiated CRC tissues. (D) Low NUCB2 expression in poorly differentiated CRC tissues. (E) H\&E staining of high expression of NUCB2 in moderately differentiated CRC. (F) High expression of NUCB2 in moderately differentiated CRC. (G) H\&E staining of the negative control of where the primary antibody was replaced by phosphate-buffered saline (H) Negative control of IHC where primary antibody was replaced by phosphate-buffered saline. CRC, colorectal cancer; NUCB2, nucleobindin 2; IHC, immunohistochemistry. All images are recorded at x200 magnification.
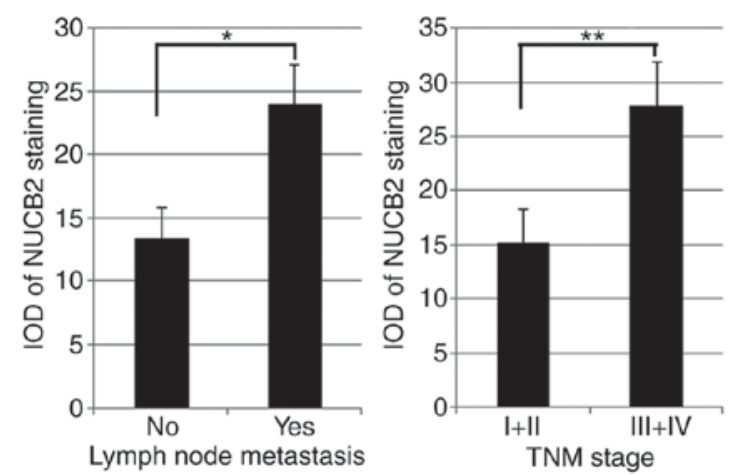

Figure 3. Quantification of NUCB2 staining in colorectal cancer tissues as detected with IOD. ${ }^{*} \mathrm{P}<0.05,{ }^{* *} \mathrm{P}<0.01$. NUCB2, nucleobindin 2 ; IOD, integrated optical density; TNM, tumor-node-metastasis.

investigated on the basis of a large clinical sample cohort. The results demonstrated an upregulation of NUCB2 in a large proportion of patients with $\mathrm{CRC}$, and elevated NUCB2 protein level was associated with the TNM stage and lymph node metastasis. These results support the hypothesis that NUCB2 may act as an oncogene in CRC with a key role in metastasis and progression.

A number of studies have also indicated an interaction between NUCB2 and the mechanistic target of rapamycin (mTOR) or AMP-activated protein kinase 
Table I. Association between NUCB2 expression and clinicopathological features of colorectal cancer.

NUCB2 expression

\begin{tabular}{|c|c|c|c|c|}
\hline & & & & \\
\hline Clinical parameters & Negative (\%) & Positive (\%) & $\chi^{2}$ & P-value \\
\hline Sex & & & 0.011 & 0.917 \\
\hline Male & $81(58.3)$ & $58(41.7)$ & & \\
\hline Female & $66(58.9)$ & $46(41.1)$ & & \\
\hline Age, years & & & 0.077 & 0.781 \\
\hline$<60$ & $37(59.7)$ & $25(40.3)$ & & \\
\hline$\geq 60$ & $109(57.7)$ & $80(42.3)$ & & \\
\hline Tumor diameter, $\mathrm{cm}$ & & & 2.522 & 0.112 \\
\hline$<20$ & $76(63.3)$ & 44 (36.7) & & \\
\hline$\geq 20$ & $70(53.4)$ & $61(46.6)$ & & \\
\hline Differentiation & & & 0.918 & 0.632 \\
\hline High & $32(64.0)$ & $18(36.0)$ & & \\
\hline Moderate & $85(56.3)$ & $66(43.7)$ & & \\
\hline Poor & $29(58.0)$ & $21(42.0)$ & & \\
\hline TNM stage & & & 6.442 & 0.011 \\
\hline $\mathrm{I}+\mathrm{II}$ & $93(65.0)$ & $50(35.0)$ & & \\
\hline $\mathrm{III}+\mathrm{IV}$ & $53(49.1)$ & $55(50.9)$ & & \\
\hline Lymph node metastasis & & & 4.088 & 0.043 \\
\hline No & $95(63.3)$ & 55 (36.7) & & \\
\hline Yes & $51(50.5)$ & $50(49.5)$ & & \\
\hline Distant metastasis & & & 2.366 & 0.171 \\
\hline No & $143(59.1)$ & $99(40.9)$ & & \\
\hline Yes & $3(33.3)$ & $6(66.7)$ & & \\
\hline Liver metastasis & & & 1.558 & 0.212 \\
\hline Negative & $144(58.8)$ & $101(41.2)$ & & \\
\hline Positive & $2(33.3)$ & $4(66.7)$ & & \\
\hline
\end{tabular}

TNM, tumor-node-metastasis; NUCB2, nucleobindin 2.

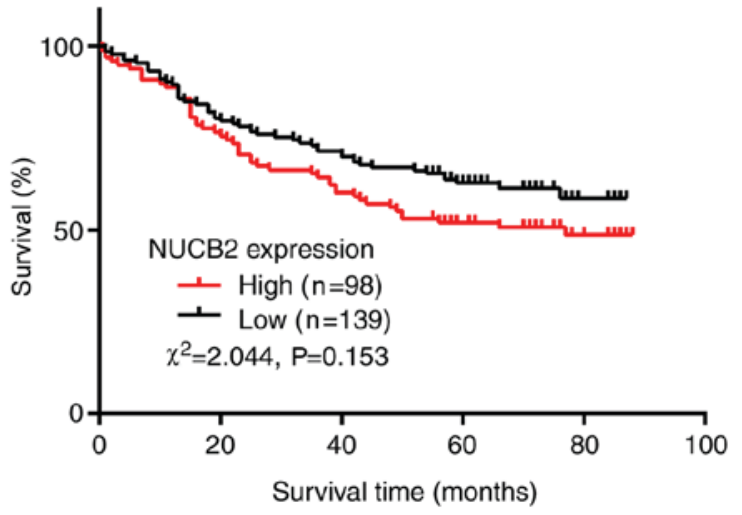

Figure 4. Kaplan-Meier survival curve analysis in patients with NUCB2 expression. There was no significant association between NUCB2 expression and overall survival $(\mathrm{P}=0.153)$. NUCB2, nucleobindin 2 .

(AMPK) pathways $(8,21)$. For example, treatment with Nestafin-1/NUCB2 enhanced the phosphorylation of AMPK and TORC2 in the rat brain (21). Kan et al (8) also indicated that NUCB2 enhanced cell migration and invasion via the liver kinase B1/AMPK/transducer of CREB protein 1/ZEB1 pathways in colon cancer. Therefore, the AMPK and mTOR pathways may serve an important role in metastasis.

A previous study by Zhang et al (7), which focused on the role of NUCB2 in cancer prognosis, demonstrated that high levels of the NUCB2 protein in prostate cancer was significantly associated with the biochemical recurrence-free survival rate, and multivariate analysis also indicated that high NUCB2 levels could be an independent prognostic factor in patients with prostate cancer. NUCB2 expression level was also reported as an independent prognostic predictor in patients with renal cell carcinoma $(9,22)$. However, in the present study, Kaplan-Meier analysis indicated no significant association between disease-free survival of patients and NUCB2 expression. Several studies have demonstrated poorer prognosis in younger patients with CRC compared with older patients $(23,24)$, whilst other studies have indicated the opposite $(25,26)$. This may be due to certain characteristics of the younger patients, which vary across different regions (27) and ethnicities (28). For instance, a higher frequency of 
colorectal neoplasia was observed among 40-49 year-old African Americans when compared with Hispanic Americans suggesting an increased susceptibility to CRC risk in this population (28). In the present study, it was determined that there was no significant association between NUCB2 expression and the age of patients with CRC as well as clinical prognosis. It is possible that the clinicopathological features of the tissue samples are responsible for this finding. Further studies are required to continuously collect more clinical data, and larger samples are also needed in order to yield more accurate and consistent results in the future.

Furthermore, in the present study, multivariate analysis demonstrated that the upregulation of NUCB2 was also not an independent prognostic predictor in patients with CRC. In view of the limitations of the present study, caused by the sample size and collection, more clinical studies are being considered for future studies, which would include a larger cohort to investigate the role of NUCB2 in CRC prognosis.

In conclusion, a significant association was detected between high NUCB2 expression level and metastasis or poor clinical outcome of CRC. This strongly indicated that NUCB2 is a cancer-associated oncogene, which is associated with aggressive progression in CRC. Additionally, NUCB2 may be useful a novel biomarker for the diagnosis, prognosis and as a potential therapeutic target for CRC. However, these results are based on a single Chinese cohort, and therefore further studies are required to validate the results.

\section{Acknowledgements}

Not applicable.

\section{Funding}

No funding received.

\section{Availability of data and materials}

All data generated or analyzed during this study are included in this published article.

\section{Authors' contributions}

WC was responsible for study conception and design and revised the manuscript, JX performed experiments and drafted the manuscript, LC analyzed the data. All authors read and approved the final manuscript.

\section{Ethics approval and consent to participate}

Approval for the present study was obtained by the Ethics Committee of the First Affiliated Hospital, Zhejiang University School of Medicine (Hangzhou, China).

\section{Consent for publication}

All patients admitted to the study provided informed consent for their participation of the present study and the publication of this data.

\section{Competing interest}

The authors declare that they have no competing interests.

\section{References}

1. Siegel RL, Miller KD and Jemal A: Cancer statistics, 2015. CA Cancer J Clin 65: 5-29, 2015.

2. Van Cutsem E and Oliveira J; ESMO Guidelines Working Group: Advanced colorectal cancer: ESMO clinical recommendations for diagnosis, treatment and follow-up. Ann Oncol 20: 61-63, 2009.

3. Yoo PS, Lopez-Soler RI, Longo WE and Cha CH: Liver resection for metastatic colorectal cancer in the age of neoadjuvant chemotherapy and bevacizumab. Clin Colorectal Cancer 6: 202-207, 2006.

4. Oh-I S, Shimizu H, Satoh T, Okada S, Adachi S, Inoue K, Eguchi $\mathrm{H}$, Yamamoto $\mathrm{M}$, Imaki $\mathrm{T}$, Hashimoto $\mathrm{K}$, et al: Identification of nesfatin-1 as a satiety molecule in the hypothalamus. Nature 443: 709-712, 2006.

5. Garcia-Galiano D, Navarro VM, Gaytan F and Tena-Sempere M: Expanding roles of NUCB2/nesfatin-1 in neuroendocrine regulation. J Mol Endocrinol 45: 281-290, 2010.

6. Zhang H, Qi C, Li L, Luo F and Xu Y: Clinical significance of NUCB2 mRNA expression in prostate cancer. J Exp Clin Cancer Res 32: 56, 2013.

7. Zhang H, Qi C, Wang A, Yao B, Li L, Wang Y and Xu Y: Prognostication of prostate cancer based on NUCB2 protein assessment: NUCB2 in prostate cancer. J Exp Clin Cancer Res 32: 77, 2013

8. Kan JY, Yen MC, Wang JY, Wu DC, Chiu YJ, Ho YW and Kuo PL: Nesfatin-1/Nucleobindin-2 enhances cell migration, invasion, and epithelial-mesenchymal transition via LKB1/AMPK/TORC1/ZEB1 pathways in colon cancer. Oncotarget 7: 31336-31349, 2016.

9. Qi C, Ma H, Zhang HT, Gao JD and Xu Y: Nucleobindin 2 expression is an independent prognostic factor for clear cell renal cell carcinoma. Histopathology 66: 650-657, 2015.

10. Suzuki S, Takagi K, Miki Y, Onodera Y, Akahira J, Ebata A, Ishida T, Watanabe M, Sasano H and Suzuki T: Nucleobindin 2 in human breast carcinoma as a potent prognostic factor. Cancer Sci 103: 136-143, 2012.

11. Ramanjaneya M, Tan BK, Rucinski M, Kawan M, Hu J, Kaur J, Patel VH, Malendowicz LK, Komarowska H, Lehnert H, et al: Nesfatin-1 inhibits proliferation and enhances apoptosis of human adrenocortical H295R cells. J Endocrinol 226: 1-11, 2015.

12. Xu Y, Pang X, Dong M, Wen F and Zhang Y: Nesfatin-1 inhibits ovarian epithelial carcinoma cell proliferation in vitro. Biochem Biophys Res Commun 440: 467-472, 2013.

13. Edge SB and Compton CC: The American Joint Committie on Cancer: The 7th edition of the AJCC cancer staging manual and the future of TNM. Ann Surg Oncol 17: 1471-1474, 2010.

14. Livak KJ and Schmittgen TD: Analysis of relative gene expression data using real-time quantitative PCR and the 2(-delta delta C(T)) method. Methods 25: 402-408, 2001.

15. Haggar FA and Boushey RP: Colorectal cancer epidemiology: Incidence, mortality, survival, and risk factors. Clin Colon Rectal Surg 22: 191-197, 2009.

16. Field K and Lipton L: Metastatic colorectal cancer-past, progress and future. World J Gastroenterol 13: 3806-3815, 2007.

17. Kraljevic Pavelic S, Sedic M, Bosnjak H, Spaventi S and Pavelic K: Metastasis: New perspectives on an old problem. Mol Cancer 10: 22, 2011.

18. Cao X, Liu XM and Zhou LH: Recent progress in research on the distribution and function of NUCB2/nesfatin-1 in peripheral tissues. Endocr J 60: 1021-1027, 2013.

19. Takagi K, Miki Y, Tanaka S, Hashimoto C, Watanabe M, Sasano H, Ito K and Suzuki T: Nucleobindin 2 (NUCB2) in human endometrial carcinoma: A potent prognostic factor associated with cell proliferation and migration. Endocr J 63: 287-299, 2016.

20. Zhang H, Qi C, Wang A, Li L and Xu Y: High expression of nucleobindin 2 mRNA: An independent prognostic factor for overall survival of patients with prostate cancer. Tumour Biol 35: 2025-2028, 2014.

21. Yang M, Zhang Z, Wang C, Li K, Li S, Boden G, Li L and Yang G: Nesfatin-1 action in the brain increases insulin sensitivity through Akt/AMPK/TORC2 pathway in diet-induced insulin resistance. Diabetes 61: 1959-1968, 2012. 
22. Fu H, Zhu Y, Wang Y, Liu Z, Zhang J, Wang Z, Xie H, Dai B, $\mathrm{Xu} \mathrm{J}$ and Ye D: High NUCB2 expression level represents an independent negative prognostic factor in Chinese cohorts of non-metastatic clear cell renal cell carcinoma patients. Oncotarget 8: 35244-35254, 2017.

23. Kaplan MA, Isikdogan A, Gumus M, Arslan UY, Geredeli C, Ozdemir N, Koca D, Dane F, Suner A, Elkiran ET, et al: Childhood, adolescents, and young adults $(\leq 25 \mathrm{y})$ colorectal cancer: Study of Anatolian Society of Medical Oncology. J Pediatr Hematol Oncol 35: 83-89, 2013.

24. Zhao L, Bao F, Yan J, Liu H, Li T, Chen H and Li G: Poor prognosis of young patients with colorectal cancer: A retrospective study. Int J Colorectal Dis 32: 1147-1156, 2017.

25. Taggarshe D, Rehil N, Sharma S, Flynn JC and Damadi A Colorectal cancer: Are the 'young' being overlooked? Am J Surg 205: 312-316, 2013.
26. Yeo SA, Chew MH, Koh PK and Tang CL: Young colorectal carcinoma patients do not have a poorer prognosis: A comparative review of 2,426 cases. Tech Coloproctol 17: 653-661, 2013.

27. You YN, Xing Y, Feig BW, Chang GJ and Cormier JN: Young-onset colorectal cancer: Is it time to pay attention? Arch Intern Med 172: 287-289, 2012.

28. Ashktorab H, Paydar M, Namin HH, Sanderson A, Begum R, Brim H, Panchal H, Lee E, Kibreab A, Nouraie M and Laiyemo AO: Prevalence of colorectal neoplasia among young African Americans and hispanic Americans. Dig Dis Sci 59: 446-450, 2014

(i) $($ ) This work is licensed under a Creative Commons Attribution-NonCommercial-NoDerivatives 4.0 International (CC BY-NC-ND 4.0) License. 